Pacific Journal of Mathematics

MAXIMAL INVARIANT SUBSPACES OF STRICTLY CYCLIC 


\title{
MAXIMAL INVARIANT SUBSPACES OF STRICTLY CYCLIC OPERATOR ALGEBRAS
}

\author{
MARY R. EMBRY
}

\begin{abstract}
A strictly cyclic operator algebra $\mathscr{A}$ on a complex Banach space $X(\operatorname{dim} X \geqq 2)$ is a uniformly closed subalgebra of $\mathscr{L}(X)$ such that $\mathscr{A} x=X$ for some $x$ in $X$. In this paper it is shown that (i) if $\mathscr{A}$ is strictly cyclic and intransitive, then $\mathscr{A}$ has a maximal (proper, closed) invariant subspace and (ii) if $A \in \mathscr{L}(X), A \neq z I$ and $\{A\}^{\prime}$ (the commutant of $A$ ) is strictly cyclic, then $A$ has a maximal hyperinvariant subspace.
\end{abstract}

1. Notation and terminology. Throughout the paper $X$ is a complex Banach space of dimension greater than one and $\mathscr{L}(X)$ is the algebra of continuous linear operators on $X$. $\mathscr{A}$ will denote a uniformly closed subalgebra of $\mathscr{L}(X)$ which is strictly cyclic and $x_{0}$ will be a strictly cyclic vector for $\mathscr{A}$ : that is, $\mathscr{A} x_{0}=X$. We do not insist that the identity element $I$ of $\mathscr{L}(X)$ be an element of $\mathscr{A}$.

If $\mathscr{B} \subset \mathscr{L}(X)$, then the commutant of $\mathscr{B}$ is $\mathscr{B}^{\prime}=\{E: E \in \mathscr{L}(X)$ and $E B=B E$ for all $B$ in $\mathscr{B}\}$. We shall use the terminology of "invariant" and "transitive" as follows: if $M \subset X$ and $\mathscr{B} \subset \mathscr{L}(X)$, then (i) $M$ is invariant under $\mathscr{B}$ if $\mathscr{B} M=\{B m: B \in \mathscr{B}$ and $m \in M\} \subset$ $M$, (ii) $M$ is an invariant subspace for $\mathscr{B}$ if $M$ is invariant under $\mathscr{B}$ and $M$ is a closed, nontrivial $(\neq\{0\}, X)$ linear subspace of $X$, (iii) $\mathscr{B}$ is transitive if $\mathscr{B}$ has no invariant subspace and intransitive if $\mathscr{B}$ has an invariant subspace. Further, if $A \in \mathscr{L}(X)$ and $\{A\}^{\prime}$ is intransitive, then each invariant subspace of $\{A\}^{\prime}$ is called a hyperinvariant subspace of $A$. Finally an invariant subspace of $\mathscr{B}$ is maximal if it is not properly contained in another invariant subspace of $\mathscr{B}$.

2. Introduction. Strictly cyclic operator algebras have been studied by A. Lambert, D. A. Herrero, and the auther of this paper. (See for example [2]-[6].) One of the major results in [2, Theorem 3.8], [3, Theorem 2], and [6, Theorem 4.5] is that a transitive subalgebra of $\mathscr{L}(X)$ containing a strictly cyclic algebra is necessarily strongly dense in $\mathscr{L}(X)$. In each of three developments the following is a key lemma: The only dense linear manifold invariant under a strictly cyclic subalgebra of $\mathscr{L}(X)$ is $X$. In Lemma 1 we shall present a generalization of this lemma which will be useful in the study of maximal invariant subspaces and noncyclic vectors of a strictly cyclic algebra $\mathscr{A}$.

Lemma 1. If $M$ is invariant under $\mathscr{A}$ and $x_{0} \in \bar{M}$, then $M=X$. 
(It should be noted that we do not require $M$ to be linear nor do we require, as was done in Lemma 3.4 of [2], that $I \in \mathscr{A}$. The proof given here is a slight modification of that given in [2].)

Proof. We shall show that $\mathscr{A} x_{0} \subset M$ and thus $X=\mathscr{A} x_{0} \subset M$. Let $\left\{x_{n}\right\}$ be a sequence in $M$ such that $\lim _{n \rightarrow \infty} x_{n}=x_{0}$. By [2, Lemma 3.1 (ii)] there exists a sequence $\left\{A_{n}\right\}$ in $\mathscr{A}$ such that $A_{n} x_{0}=x_{0}-x_{n}$ and $\lim _{n \rightarrow \infty}\left\|A_{n}\right\|=0$. Thus for $n$ sufficiently large, $\left\|A_{n}\right\|<1$ and $\left(I-A_{n}\right)^{-1}=\sum_{k=0}^{\infty}\left(A_{n}\right)^{k}$. Consequently, $\mathscr{A}\left(I-A_{n}\right)^{-1} \subset \mathscr{A}$ and since $x_{0}=\left(I-A_{n}\right)^{-1} x_{n}$, we have $\mathscr{A} x_{0}=\mathscr{A}\left(I-A_{n}\right)^{-1} x_{n} \subset \mathscr{A} x_{n} \subset M$, as desired.

For the sake of future reference we restate and reprove the transitivity theorem.

THEOREM 1. If $\mathscr{A}$ is a strictly cyclic transitive subalgebra of $\mathscr{L}(X)$, then $\mathscr{A}$ is strongly dense in $\mathscr{L}(X)$.

Proof. Using Lemma 1 we can show (as in [2, Lemma 3.5]) that each densely defined linear transformation commuting with $\mathscr{A}$ is everywhere defined and continuous. Further, again using Lemma 1, we can show that if $E \in \mathscr{A}$ and $z \in \sigma(E)$, then either $z I-E$ is not one-to-one or does not have dense range. Thus if $\mathscr{A}$ is transitive, necessarily $E=z I$. Consequently, it follows from [1, p. 636 and Cor. 2.5, p. 641] that $\mathscr{A}$ is strongly dense in $\mathscr{L}(X$.

3. Maximal invariant subspaces. In [2, Theorem 3.1] it is shown that every strictly cyclic, separated operator algebra $\mathscr{A}$ has a maximal invariant subspace. ( $\mathscr{A}$ is separated by $x_{0}$ if $A=0$ whenever $A \in \mathscr{A}$ and $A x_{0}=0$.) Theorem 2 allows us to obtain the same result without the hypothesis that $\mathscr{A}$ be separated, provided $\mathscr{A}$ is intransitive.

THEOREM 2. An intransitive, strictly cyclic subalgebra $\mathscr{A}$ of $\mathscr{L}(X)$ has a maximal invariant subspace.

Proof. Let $\mathscr{C}=\{M: M$ is an invariant subspace of $\mathscr{A}\}$. By hypothesis $\mathscr{l} \neq \varnothing$. We shall order $\mathscr{C l}$ by set inclusion and show that each linearly ordered subset of $\mathscr{C}$ has an upper bound in $\mathscr{C}$. To this end we let $\left\{M_{\alpha}\right\}$ be a linearly ordered subset of $\mathscr{M}$. Then $\mathrm{U}_{\alpha} M_{\alpha}$ is invariant under $\mathscr{C}$. By Lemma 1 , if $\overline{\mathrm{U}_{\alpha} M_{\alpha}}=X$, then $\mathrm{U}_{\alpha} M_{\alpha}=X$ and consequently $x_{0} \in M_{\alpha}$ for some value of $\alpha$. Since this last implies that $X=\mathscr{A} x_{0} \subset \mathscr{A} M_{\alpha} \subset M_{\alpha}$ and contradicts the fact that $M_{\alpha}$ is a proper closed linear subspace of $X$, we see that $\mathrm{U}_{\alpha} M_{\alpha}$ is not 
dense in $X$. Thus $\overline{\mathrm{U}_{\alpha} M_{\alpha}}$ is an element of $\mathscr{M}$ and is an upper bound for $\left\{M_{\alpha}\right\}$. By the Maximality Principle $\mathscr{C}$ has a maximal element.

Lemma 1 and the Maximality Principle can be combined to arrive at other similar results. For example, (i) if $\mathscr{A}$ is intransitive and strictly cyclic, then $\mathscr{A}$ has a proper maximal invariant subset (this will be discussed further in $\S 4$ ) and (ii) if $X$ is a Hilbert space and $\mathscr{A}$ has a reducing subspace (that is, an invariant subspace of $\mathscr{A}$ which is also invariant under $\left.\mathscr{A}^{*}=\left\{A^{*}: A \in \mathscr{A}\right\}\right)$, then $\mathscr{A}$ has a maximal reducing subspace.

In [2, Theorem 3.7] it is shown that if $A$ is not a scalar multiple of $I$ and $\{A\}^{\prime}$ is strictly cyclic, then $A$ has a hyperinvariant subspace. This result combined with Theorem 2 yields the following:

COROLlary 1. If $A$ is not a scalar multiple of $I$ and $\{A\}^{\prime}$ is strictly cyclic, then $A$ has a maximal hyperinvariant subspace.

We shall now turn our attention to intransitive, strictly cyclic operator algebras on a Hilbert space $X$. If $M$ is a closed linear subspace of $X, P_{M}$ will denote the orthogonal projection of $X$ onto $M$ and $M^{\perp}$ the orthogonal complement of $M: M^{\perp}=\{y:\langle y, m\rangle=0$ for all $m$ in $M\}$. Furthermore, $\mathscr{A}^{*}=\left\{A^{*}: A \in \mathscr{A}\right\}$.

In the Hilbert space situation we are able to conclude that $\mathscr{A}^{*} / M$ is strongly dense in $\mathscr{L}\left(M^{\perp}\right)$ when $M$ is a maximal invariant subspace for $\mathscr{A}$. This remains an open question if $X$ is an arbitrary Banach space and is a particularly interesting one if $X$ is reflexive. For in that case if $M$ is a maximal invariant subspace of $\mathscr{A}$, then $M^{\perp}=$ $\left\{x^{*}: x^{*}(M)=0\right\}$ is a minimal invariant subspace of $\mathscr{A}^{*}$.

THEOREM 3. Let $\mathscr{A}$ be a strictly cyclic operator algebra on a Hilbert space $X$. If $M$ is a maximal invariant subspace of $\mathscr{A}$, then

(i ) $\left(I-P_{M}\right) \mathscr{A}\left(I-P_{M}\right) x_{0}=M^{\perp}$ and (ii) $\mathscr{A}^{*}\left(I-P_{M}\right)$ is strongly dense in $\mathscr{L}\left(M^{\perp}\right)$.

Proof. Note first that $\left(I-P_{M}\right) \mathscr{A}\left(I-P_{M}\right)=\left(I-P_{M}\right) \mathscr{A}$, so that (i) is immediate. Since $M$ is a maximal invariant subspace for $\mathscr{A}, M^{\perp}$ is a minimal invariant subspace for $\mathscr{A}^{*}$. Thus each of $\mathscr{A}^{*}\left(I-P_{M}\right)$ and $\left(I-P_{M}\right) \mathscr{A}\left(I-P_{M}\right)$ is transitive on $M^{\perp}$. Thus the uniform closure of $\left(I-P_{M}\right) \mathscr{A}\left(I-P_{M}\right)$ in $\mathscr{L}\left(M^{\lrcorner}\right)$is transitive and by (i) is strictly cyclic; hence by Theorem $1\left(I-P_{M}\right) \mathscr{A}\left(I-P_{M}\right)$ is strongly dense in $\mathscr{L}(M)$, which concludes our proof of (ii).

Theorem 4. Let $X$ be a Hilbert space, $A \in \mathscr{L}(X)$ and $\{A\}^{\prime}$ strictly cyclic. If $M$ is a maximal invariant subspace for $\{A\}^{\prime}$, then there exists a multiplicative linear functional $f$ on $\{A\}^{\prime \prime}$ such 
that for each $E$ in $\{A\}^{\prime \prime},(E-f(E) I)(X) \subset M$.

Proof. As we noted in the proof of Theorem 3,

$$
\mathscr{B}=\left(I-P_{M}\right)\{A\}^{\prime}\left(I-P_{M}\right)
$$

is strongly dense in $\mathscr{L}\left(M^{\lrcorner}\right)$and thus its commutant consists of the scalar multiples of the identity operator on $M^{\perp}$. Since $\{A\}^{\prime \prime} \subset\{A\}^{\prime}$ and $M$ is invariant under $\{A\}^{\prime}$, we know that $\left(I-P_{M}\right)\{A\}^{\prime \prime}\left(I-P_{M}\right)$ is contained in the commutant of $\mathscr{B}$ on $M^{\perp}$ and hence $\left(I-P_{M}\right)\{A\}^{\prime \prime}(I-$ $\left.P_{M}\right) \subset\left\{z\left(I-P_{M}\right)\right\}$. Thus for $E$ in $\{A\}^{\prime \prime}$, there exists a complex number $z$ such that $\left(I-P_{M}\right) E\left(I-P_{M}\right)=z\left(I-P_{M}\right)$. Therefore, $\left(I-P_{M}\right)(E-$ $z I)=0$ since $M$ is invariant under $\{A\}^{\prime \prime}$; or equivalently $(E-z I)(X) \subset$ $M$. Since $M$ is a proper subset of $X$, it is now obvious that the number $z$ for which $(E-z I)(X) \subset M$ is unique. Define $f(E)=z$.

That $f$ is linear follows immediately from the fact that $f(E)$ is the unique number for which $(E-f(E) I)(X) \subset M$. Furthermore, since $M$ is invariant under $\{A\}^{\prime \prime},(F E-f(E) F)(X) \subset M$ for all $E, F \in\{A\}^{\prime \prime}$. Consequently (by uniqueness again), $0=f(F E-f(E) F)=f(F E)-$ $f(E) f(F)$ and thus we see that $f$ is multiplicative.

Corollary 2. Let $A \in \mathscr{L}(X)$ where $X$ is a Hilbert space. If the range of $A-z I$ is dense in $X$ for each complex $z$, then $\{A\}^{\prime}$ is not strictly cyclic.

Proof. Except for one minor technicality, Corollary 2 follows immediately from Theorem 4 . For, if $\{A\}^{\prime}$ is strictly cyclic and intransitive, by Theorem 4 there exists a complex number $f(A)$ such that the range of $A-f(A) I$ is contained in a proper subspace of $X$. By Corollary 1 the only other way in which $\{A\}^{\prime}$ can be strictly cyclic is when $A=z I$ for some complex $z$, in which case the range of $A-$ $z I$ is certainly not dense in $X$.

In [2, Lemma 3.6] and [3, Proposition 2], it is shown that if $E \in \mathscr{A}$,' where $\mathscr{A}$ is strictly cyclic and $z \in \sigma(E)$, then either $z I-E$ is not one-to-one or $z I-E$ does not have dense range. Corollary 2 now adds to our knowledge of $\sigma(A)$ where $\{A\}^{\prime}$ is strictly cyclic: in this case we know that for at least one value of $z$, the range of $A-z I$ is nondense. Indeed we have the stronger result:

Corollary 3. Let $A \in \mathscr{L}(X)$ where $X$ is a Hilbert space. If $\{A\}^{\prime}$ is strictly cyclic, then there exists a common eigenvector for $\left\{A^{*}\right\}^{\prime \prime}$.

Proof. The case in which $\{A\}^{\prime}=\mathscr{L}(X)$ is trivial. Thus we assume $A \neq z I$. By Theorem 4 if $E \in\{A\}^{\prime \prime}$, there exists a complex number $f(E)$ such that $(E-f(E) I)(X) \subset M$ where $M$ is a maximal 
invariant subspace of $\{A\}^{\prime}$. Therefore, $E^{*}\left(I-P_{M}\right) x_{0}=f(E)^{*}\left(I-P_{M}\right) x_{0}$ and $\left(I-P_{M}\right) x_{0} \neq 0$ since $x_{0}$ is cyclic for $\{A\}^{\prime}$ and $M$ is a proper invariant subspace for $\{A\}^{\prime}$.

4. Noncyclic vectors of $\mathscr{A}$. In this last section of this paper we shall discuss briefly several properties of the set of noncyclic vectors of a strictly cyclic operator algebra $\mathscr{A}$. A vector $x$ is noncyclic for $\mathscr{A}$ if $\mathscr{A} x$ is not dense in $X$. These results are summarized in Theorem 5. Parts (i) and (iii) of Theorem 5 also are found in [5, Theorem 2].

THEOREM 5. Let $N$ be the set of noncyclic vectors of a strictly cyclic operator algebra $\mathscr{A}$,

(i) if $x \notin N$, then $x$ is a strictly cyclic vector for $\mathscr{A}$,

(ii) $N$ is invariant under $\mathscr{A}$,

(iii) $N$ is closed in $X$,

(iv) $N$ is the unique proper maximal invariant subset of $\mathscr{A}$,

(v) if $N$ is not linear, then $N+N=X$, where $N+N=\{x+$ $y: x, y \in N\}$.

Proof. (i) If $x \notin N$, then $\overline{\mathscr{A} x}=X$ and thus by Lemma 1 since $\mathscr{A} x$ is invariant under $\mathscr{A}$, we have $\mathscr{A} x=X$ and $x$ is strictly cyclic. (ii) Assume that $x \in N$ and $A \in \mathscr{A}$. Then $\mathscr{A} A x \subset \mathscr{A} x$ and consequently $\mathscr{A} A x \neq X$. That is, $A x \in N$ for each $A$ in $\mathscr{A}$ which proves (ii). (iii) $\mathrm{By}$ (ii) $\mathscr{A} \mathrm{N} \subset N$. Since $\mathscr{A}$ has a strictly cyclic vector, we know by Lemma 1 that $\bar{N}$ contains no strictly cyclic vector for $\mathscr{A}$. Thus by (i) $\bar{N}$ contains only noncyclic vectors for $\mathscr{A}$, which says that $N$ is closed. (iv) By (ii) $N$ is invariant under $\mathscr{A}$. By hypothesis $\mathscr{A}$ has a strictly cyclic vector so that $N \neq X$. These two observations essentially prove (iv) since an element $x$ of a proper invariant subset of $\mathscr{A}$ is necessarily an element of $N$. (v) If $N$ is nonlinear, then since $N$ is homogeneous, we know that $N \neq N+N$. Therefore, since $N+N$ is invariant under $\mathscr{A}$ (by (ii) we know that $N+N=X$ by (iv)).

To see that there exist strictly cyclic operator algebras for which $N$ is linear and those for which $N$ is nonlinear let us reconsider Example 1 of [2].

ExAmple. Let $X$ be a Banach space, $\operatorname{dim} X \geqq 2$ and let $x_{0} \in X$, $x_{0} \neq 0$. Let each of $x^{*}$ and $y^{*}$ be a continuous linear functional on $X$ such that $x^{*}\left(x_{0}\right)=y^{*}\left(x_{0}\right)=1$. For each $x$ in $X$ define $A_{x}$ by

$$
A_{x} y=x^{*}(x)\left[y-y^{*}(y) x_{0}\right]+y^{*}(y) x
$$


and let $\mathscr{A}=\left\{A_{x}: x \in X\right\}$.

It was observed in [2] that $\mathscr{A}$ is a strictly cyclic operator algebra with strictly cyclic, separating vector $x_{0}$.

A simple argument shows that a vector $y_{0}$ of $X$ is cyclic (and hence by Theorem 5 strictly cyclic) if and only if $y^{*}\left(y_{0}\right) \neq 0$ and $x^{*}\left(y_{0}\right) \neq$ 0 . Thus the set $N$ of noncyclic vectors coincides with $\operatorname{ker} y^{*} \cup \operatorname{ker} x^{*}$. Consequently, $N$ is linear if $x^{*}$ and $y^{*}$ are dependent and nonlinear otherwise.

\section{REFERENCES}

1. W. B. Arveson, A density theorem for operator algebras, Duke Math. J., 34 (1967), 635-647.

2. M. R. Embry, Strictly cyclic operator algebras on a Banach space, Pacific J. Math., 45 (1973), 443-452.

3. D. A. Herrero, Algebras de Operadores Transitivas que Contienen una Subalgebra de Multiplicidad Estricta Finita, Revista de la Union Mat. Argentina, (to appear).

4. - Operator algebras of finite strict multiplicity, Indiana Univ. Math. J., 22 (1972), 13-24.

5. D. A. Herrero and A. Lambert, On Strictly Cyclic Algebras, P-Algebras and Reflexive Operators, preprint.

6. A. Lambert, Strictly cyclic operator algebras, Pacific J. Math., 39 (1971), 717-726.

Received July 14, 1972 and in revised form August 25, 1972.

University of North Carolina at Charlotte 


\section{PACIFIC JOURNAL OF MATHEMATICS}

\section{EDITORS}

RICHARD ARENS (Managing Editor) University of California

Los Angeles, California 90024

R. A. Beaumon'T

University of Washington Seattle, Washington 98105
J. Dugundj1*

Department of Mathematics University of Southern California Los Angeles, California 90007

D. Gilbarg and J. Milgram Stanford University Stanford, California 94305

\section{ASSOCIATE EDITORS}

E. F. BECKENBACH

B. H. NeUMANN

F. WOLF

K. YOSHIDA

\section{SUPPORTING INSTITUTIONS}

UNIVERSITY OF BRITISH COLUMBIA CALIFORNIA INSTITUTE OF TECHNOLOGY UNIVERSITY OF CALIFORNIA MONTANA STATE UNIVERSITY UNIVERSITY OF NEVADA NEW MEXICO STATE UNIVERSITY OREGON STATE UNIVERSITY UNIVERSITY OF OREGON OSAKA UNIVERSITY

\section{UNIVERSITY OF SOUTHERN CALIFORNIA STANFORD UNIVERSITY UNIVERSITY OF TOKYO UNIVERSITY OF UTAH WASHINGTON STATE UNIVERSITY UNIVERSITY OF WASHINGTON AMERICAN MATHEMATICAL SOCIETY NAVAL WEAPONS CENTER}

* C. R. DePrima California Institute of Technology, Pasadena, CA 91109, will replace J. Dugundji until August 1974. 


\section{Pacific Journal of Mathematics}

\section{Vol. 49, No. $1 \quad$ May, 1973}

A. Bigard, Free lattice-ordered modules ...........................

Richard Bolstein and Warren R. Wogen, Subnormal operators in strictly cyclic

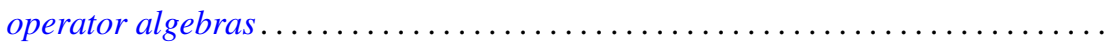

Herbert Busemann and Donald E. Glassco, II, Irreducible sums of simple

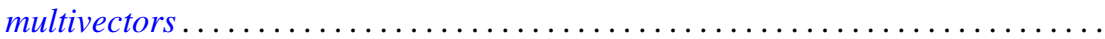

W. Wistar (William) Comfort and Victor Harold Saks, Countably compact groups

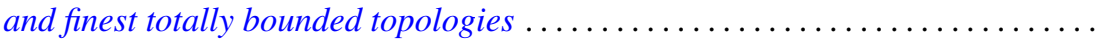

Mary Rodriguez Embry, Maximal invariant subspaces of strictly cyclic operator

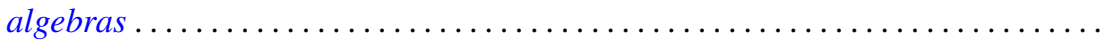

Ralph S. Freese and James Bryant Nation, Congruence lattices of semilattices......

Ervin Fried and George Grätzer, A nonassociative extension of the class of

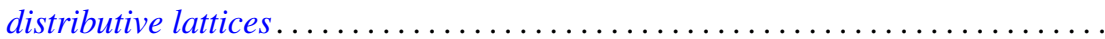

John R. Giles and Donald Otto Koehler, On numerical ranges of elements of locally

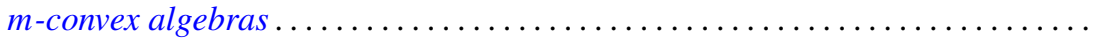

David A. Hill, On dominant and codominant dimension of $\mathrm{QF}-3$ rings ........ John Sollion Hsia and Robert Paul Johnson, Round and Pfister forms over $R(t) \ldots$ I. Martin (Irving) Isaacs, Equally partitioned groups . . . . . . . . . . . . . .

Athanassios G. Kartsatos and Edward Barry Saff, Hyperpolynomial approximation of solutions of nonlinear integro-differential equations.

Shin'ichi Kinoshita, On elementary ideals of $\theta$-curves in the 3-sphere and 2-links in the 4-sphere

Ronald Brian Kirk, Convergence of Baire measures

R. J. Knill, The Seifert and Van Kampen theorem via regular covering spaces ..

Amos A. Kovacs, Homomorphisms of matrix rings into matrix rings ..

Young K. Kwon, HD-minimal but no $H D$-minimal ..........

Makoto Maejima, On the renewal function when some of the mean renewal lifetimes are infinite

Juan José Martínez, Cohomological dimension of discrete modules over profinite groups.

W. K. Nicholson, Semiperfect rings with abelian group of units

Louis Jackson Ratliff, Jr., Three theorems on imbedded prime divisors of principal ideals.

Billy E. Rhoades and Albert Wilansky, Some commutants in $B(c)$ which are almost matrices

John Philip Riley Jr., Cross-sections of decompositions . . .

Keith Duncan Stroyan, A characterization of the Mackey uniformity $m\left(L^{\infty}, L^{1}\right)$ for

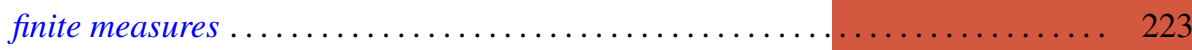

Edward G. Thurber, The Scholz-Brauer problem on addition chains . . . . . . . . . 229

Joze Vrabec, Submanifolds of acyclic 3-manifolds ............

Philip William Walker, Adjoint boundary value problems for compactified singular

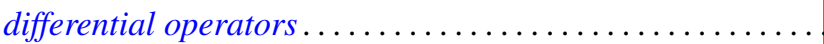

